Animal Study

\title{
Pulsed Radiofrequency on Dorsal Root Ganglion Relieved Neuropathic Pain Associated with Downregulation of the Spinal Interferon Regulatory Factor 8, Microglia, p38MAPK Expression in a CCI Rat Model
}

Rongguo Liu, MD, PhD¹, Xueru Xu, MD¹, Yunlu Xu, PhD², Xiangyu Fang, MD¹, and Xingwu Lin, MD'

From: ${ }^{1}$ Department of Pain Management, Fujian Provincial Hospital, Fujian Key Laboratory of Geriatrics, Provincial Clinic College of Fujian Medical University, Fuzhou, Fujian, China; ${ }^{2}$ Department of Pharmacology, College of Pharmacology, Fujian Medical University, Fuzhou, Fujian,

China

Address Correspondence: Ronggue Liu, MD, PhD Department of Pain Management, Provincial Clinic

College of Fujian Medical University

134 Dongjie Rd, Fuzhou 350001, Fujian, China E-mail: Irgfw88@sina.com

Disclaimer: This study was supported by Project of Medical Innovation of Fujian Province (2016-CX-3). Conflict of interest: Each author certifies that he or she, or a member of his or her immediate family, has no commercial association

(i.e., consultancies, stock ownership, equity interest, patent/licensing arrangements, etc.) that might pose a conflict

of interest in connection with the submitted manuscript.

Manuscript received: 06-04-2017

Revised manuscript received: 12-03-2017 Accepted for publication: 01-11-2018

Free full manuscript: www.painphysicianjournal.com
Background: Interferon regulatory factor 8 (IRF8), which is induced by peripheral nerve injury (PNI), plays a key role in activating spinal microglia to release inflammatory cytokines in a p38dependent way, thereafter results in formation of central sensitization. Pulsed radiofrequency (PRF) on dorsal root ganglion (DRG) alleviates neuropathic pain and inhibits the microglial activation in chronic constriction injury $(\mathrm{CCl})$ rats. However, the consequences of PRF on spinal IRF8 of $\mathrm{CCI}$ rats remains unknown.

Objectives: We explore if PRF on DRG of rats with $\mathrm{CCl}$ could restrain IRF8, microglia, and p38 hyperactivity in the spinal cord to alleviate neuropathic pain.

Study Design: A randomized, controlled animal study.

Setting: Department of Pain Management, Fujian Provincial Hospital, Fujian Key Laboratory of Geriatrics, Provincial Clinic College of Fujian Medical University.

Methods: The changes in pain behaviors and the expressions of IRF8, Iba1 and p-p38 in the spinal cord of $\mathrm{CCl}$ rats which were administrated with antisense/ mismatch oligodeoxynucleotide of IRF8 were studied. Rats in $\mathrm{CCl}+\mathrm{AS}$ ODN group, $\mathrm{CCl}+\mathrm{MM}$ ODN group or $\mathrm{CCl}+\mathrm{NS}$ group were intrathecally treated with antisense oligodeoxynucleotide of IRF8, mismatch oligodeoxynucleotide of IRF8 or same volume $0.9 \% \mathrm{NaCl}$ once daily respectively, beginning from the day after nerve transection 12 hours and lasting for 7 days. The effects of PRF on L4-5 DRG of rats with CCI were investigated. PRF was applied adjacent to the L4-5 DRG at an intensity of $45 \mathrm{~V}$ for 6 minutes after $\mathrm{CCl}$, whereas the control rats were treated without radiofrequency current. The withdrawal thresholds were studied and the spinal levels of IRF8, ionized calcium-binding adapter molecule 1 (Iba1, microglia characteristic marker) and p-p38 were calculated by ELISA, western blot, RT-PCR, and immunofluorescence.

Results: Intrathecal administration of antisense oligodeoxynucleotide of IRF8 led to the reversal of CCl-induced allodynia, lower activation of spinal microglia and p-p38. Withdrawal thresholds were partially recovered after a single PRF treatment for 14 days. CCl-induced IRF8 upregulation, microglia hyperactivity, and p38 phosphorylation in the spinal cord were reduced due to PRF treatment. However, PRF did not alter pain behaviors and pain signals in normal rats.

Limitations: In our study, one time point was selected just to assess the levels of microglia, and p-p38. The changes of IRF8, microglia, p-p38 in the ipsilateral DRG were not investigated. A more detailed study on how PRF on the DRG could further relieve NP is needed.

Conclusions: Restraining IRF8, microglia and p38 hyperactivity in the spinal cord of $\mathrm{CCl}$ rats involved in the contribution to the long-lasting analgesia of PRF.

Keywords: Neuropathic pain, pulsed radiofrequency, dorsal root ganglion, microglia, p38MAPK, Interferon regulatory factor 8 , chronic constriction injury of sciatic nerve

Pain Physician 2018; 21:E307-E322 
$\mathrm{N}$ europathic pain (NP) has been defined as "pain arising as a direct consequence of a lesion or disease affecting the somatosensory system" (1) resulting from several idiopathic conditions, such as diabetes, herpes zoster infection, and trauma. NP adversely affects the somatic, psychological, and sociological health of patients. It remains hard to treat due to the complicated etiology and mechanisms that include several neurotransmitter systems, receptors, ionic channels, and cell types $(2,3)$. Thus, current pharmacotherapy rarely resolves intractable pain in patients. Recently, pulsed radiofrequency (PRF) on the dorsal root ganglion(DRG) has been proved to significantly alleviate neuropathic pain (4-6). PRF has been increasingly accepted due to its lack of destructive effects on neurons and pain relief efficacy in several types of chronic pain. Although, the mechanisms by which PRF on DRG reduces the pain remain to be elucidated.

Recently, PRF was administrated on DRG in rats with peripheral nerve injury (PNI) to downregulate microglial activation in the spinal cord and improve pain behaviors $(7,8)$. Microglia are the resident macrophages in central nervous system (CNS) and they react to the stimuli that may affect homeostasis and induce pathological alterations (9-11). Microglia was induced by PNI to release inflammatory cytokines, such as IL-1 $\beta$, IL-6, IL-18, TNF- $\alpha$, and chemokines in a p38-dependent way. The activated microglia could induce central sensitization and maintain microglia-related persistent pain (12-15). In addition, Masuda et al (16) reported that interferon regulatory factor 8 (IRF8) was significantly enhanced in the microglia after PNI and played a pivotal role in activating gene expressions that transform microglia into a reactive phenotype.

In this study, we intrathecally administered IRF8 antisense oligodeoxynucleotide in the early phase of $\mathrm{CCl}$ to further affirm the function of IRF8 in neuropathic pain. We also investigated whether PRF on the DRG of $\mathrm{CCl}$ rats could suppress IRF8, microglia and p38 hyperactivity in the spinal cord to alleviate neuropathic pain.

\section{Methods}

\section{Animals and Materials}

Male Sprague Dawley (SD) rats (4 months old, 250-280 g) were obtained from the Experimental Animal Center of Fujian Medical University, Fuzhou. Animals were housed under a $12 \mathrm{~h}$ light-dark cycle at 22-24 ${ }^{\circ} \mathrm{C}$ with ad libitum access to food and water in the Pharmacy College of Fujian Medical University (SPF class). All procedures in this study were approved by the Fujian Medical University Experimental Animal Welfare Ethics Committee.

\section{Study Design}

Four hundred and eight male SD rats were randomly (according to the method of random number table) divided into 8 groups: Sham group, $\mathrm{CCl}$ group, $\mathrm{CCl}+\mathrm{PRF}$ group, $\mathrm{CCl}+$ Sham-PRF (CCl + SPRF) group, Sham + PRF group, $\mathrm{CCl}$ with IRF8 antisense oligodeoxynucleotide $(\mathrm{CCl}+\mathrm{AS} \mathrm{ODN})$ group, $\mathrm{CCl}$ with IRF8 mismatch oligodeoxynucleotide (CCl + MM ODN) group, $\mathrm{CCl}$ with normal saline $(\mathrm{CCl}+\mathrm{NS})$ group. Each group contained 51 rats.

\section{Effects of IRF8 Antisense Oligodeoxynucleotide on IRF8, Microglia and p38 Hyperactivity}

Rats in the following 5 groups (Sham, $\mathrm{CCl}, \mathrm{CCl}+\mathrm{AS}$ ODN, CCl+MM ODN, and CCl+NS groups) were studied. All rats (except in the Sham group) were subject to $\mathrm{CCl}$ of the right sciatic nerve. The rats in $\mathrm{CCl}+\mathrm{AS}$ ODN group, $\mathrm{CCl}+\mathrm{MM}$ ODN group and $\mathrm{CCl}+\mathrm{NS}$ group were intrathecally treated with antisense ODN of IRF8, mismatch ODN of IRF8 and same volume $0.9 \% \mathrm{NaCl}$, respectively. Pain behaviors and the levels of IRF8, Iba1 and p-p38 in the spinal cord were assayed and compared among the 5 groups.

\section{PRF Effects on Neuropathic Pain and the Spinal IRF8, Microglia and p38 Hyperactivity}

Four groups were observed, including Sham, CCl, $\mathrm{CCl}+\mathrm{PRF}, \mathrm{CCl}+\mathrm{SPRF}$. All rats (except in the sham group) were subject to $\mathrm{CCl}$ of the right sciatic nerve. On the postoperative day 7, PRF was applied to the ipsilateral L4-5 DRG in CCl + PRF group, CCl + SPRF group was kept design as control. Pain behaviors and the expressions of IRF8, Iba1, p-p38 were measured.

\section{PRF Therapy on Normal Rats}

Rats in the 2 groups, including the Sham and Sham + PRF groups were studied. PRF was applied to the ipsilateral L4-5 DRG in Sham + PRF group. Pain behavioral changes and the expressions of IRF8, Iba1, and p-p38 were measured in the spinal cord.

All changes of mechanical allodynia and thermal hyperalgesia were studied before the operation (D0), on first (D01), third (D03), fifth (D05), and seventh (D07) postoperative day and first (D1), third (D3), fifth (D5), seventh (D7), ninth (D9), eleventh (D11), and fourteenth (D14) day after PRF treatment or completion of intrathe- 
cal injection. The spinal IRF8 levels were measured by western blot, RT-PCR, and immunofluorescence on D14, by ELISA on D0, D01, D03, D05, D07, D1, D3, D5, D7, D9, D11, D14. The spinal Iba1 and p-p38 expression were assayed by western blot, RT-PCR, and immunofluorescence on D14. Three rats have been used by ELISA in per timepoint per group, 5 rats have been used by western blot, RT-PCR, and immunofluorescence, respectively.

\section{Neuropathic Pain Model}

PNI was performed according to the $\mathrm{CCl}$ model as described previously (17). Briefly, the rat's right common sciatic nerve was exposed at the mid-thigh level under anesthesia and 4 loose ligatures (4-0 chromic catgut) were tied around the nerve (injured hind paw). The muscle and skin were then closed in layers. In the Sham group, the right common sciatic nerve was exposed, but no ligation was carried out. All operations were performed by the same experimenter.

\section{Intrathecal Administration}

The rats received intrathecal catheter implantation before $\mathrm{CCl}$. Rats were anesthetized with an intraperitoneal injection of $10 \%$ chloral hydrate $(300 \mathrm{mg} / \mathrm{kg})$. A PE-10 polyethylene catheter was implanted between the L4 and L5 vertebrae to reach the subarachnoid space of the spinal cord (18). The outer part of the catheter was plugged and fixed onto the skin upon wound closure. Rats showed neurological deficits after the catheter implantation were euthanized. Antisense oligodeoxynucleotide (AS ODN) of IRF8(5ug/5ul, Biosune, Shanghai, China) and mismatch ODN (MM ODN) of IRF8 (5ug/5ul, Biosune, Shanghai, China) were dissolved in sterile $0.9 \%$ saline, the rats were intrathecally treated with antisense ODN of IRF8, mismatch ODN of IRF8 or equivalent volume $0.9 \%$ saline once daily for 7 days, 12 hours after nerve injury. Drugs or vehicles were intrathecally injected via the implanted catheter in a 10 $\mu \mathrm{l}$ volume of solution followed by $10 \mu \mathrm{l}$ of vehicle for flushing. Each injection lasted at least 5 minutes. After injection, the needle remained in situ for 2 minutes before being withdrawn.

\section{Pulsed Radiofrequency Treatment}

On the postoperative day 7, the right L4-5 DRG of rats were exposed in $\mathrm{CCl}+\mathrm{SPRF}$, Sham + PRF and $\mathrm{CCl}$ + PRF groups through laminectomy and facetectomy, without injury to the duramater. PRF was then applied to the corresponding DRG, using PRF therapeutic equipment (Cosman RFG-4 Generator, Burlington, MA, USA), by placing the active tip electrode perpendicularly adjacent to the DRG. The motor stimulation test was used instead of the sensory stimulation test. PRF waves were applied after carrying out the motor stimulation test, via muscle contraction of the lower extremities. Stimulation parameters of PRF waves were set as follows: 2 bursts $/ \mathrm{s}$, duration $=20$ milliseconds, output voltage $=$ $45 \mathrm{~V}$, maximum temperature $=42^{\circ} \mathrm{C}$, and the stimulated time $=6$ minutes. No radiofrequency stimulation was done to the DRG in the $\mathrm{CCI}+$ SPRF control group.

\section{Behavioral Testing}

\section{Mechanical Hypersensitivity}

Rats were placed in a plastic chamber $(20 \mathrm{~cm} \times 25$ $\mathrm{cm} \times 15 \mathrm{~cm}$ ) and habituated for 15 minutes before testing. Paw withdrawal threshold (PWT) was evaluated with von Frey filaments (Stoelting, Wood Dale, IL, USA) using the previously described up-down method (19). Each filament was applied perpendicularly to the ipsilateral territory, near the center of the vibrissal pad. Avoiding further contact with filament, quickly turning head away, scratching the stimulated area, or attacking the filament was considered a positive response. An allodynic rat was defined as the one where $50 \%$ PWT is $<4.0 \mathrm{~g}$ (withdrawal in response to non-noxious tactile stimulus).

\section{Thermal Hyperalgesia}

Paw withdrawal latency (PWL) was tested by measuring the withdrawal response of the hind paw to heat stimulation with the Plantar Test Apparatus (TaiMeng science and technology, Chengdu, China) as described by Hargreaves et al (20) The cut-off latency was 30 seconds to avoid thermal injury. The withdrawal latency at each time point was an average of 3 latencies separated by an interval of 5 minutes. The tests were conducted on the same days as the von Frey test, and both tests were conducted by the same experimenter who was blind to the group allocation of the rats.

\section{ELISA}

The spinal cord of lumbar (L4-L5) ipsilateral quadrant to the lesion was collected. Samples were added PBS (pH7.4), homogenized by hand or grinders, centrifuged for 20 minutes at the speed of 3000 r.p.m. and then supernatant was removed. The levels of IRF8 were determined by Rat Interferon Regulatory Factor 8 ELISA Kit (Luyuan Dade biological technology, China) with a commercial reagent kit following the manufacturer's instruction. 


\section{Western Blot}

The spinal cord of lumbar (L4-L5) ipsilateral quadrant to the lesion was collected. Tissues were homogenized and centrifuged at $5000 \times \mathrm{g}$ for 10 minutes at $4^{\circ} \mathrm{C}$ and protein concentrations were measured using a Bio-Rad DC Protein Assay Kit. Equal amounts of protein were resolved by $10 \%$ SDS-PAGE and transferred to PVDF membranes (Millipore, Bedford, MA, USA). The membranes were blocked with $5 \%$ non-fat milk at room temperature and incubated overnight at $4^{\circ} \mathrm{C}$ with primary antibodies (rabbit anti-IRF8, \#5628, 1:1000, Abcam, USA; rabbit anti-lba1, ab178847, 1:1000, Abcam, USA; rabbit anti-phosphorylated-p38MAPK (Thr180/ Tyr182), \#4631, 1:1000, Cell Signaling Technology, USA; rabbit anti- $\beta$-actin, \#4967, 1:1000, Cell Signaling Technology, USA.). Then, the membranes were incubated with HRP conjugated secondary antibody (1:5000, Thermo Scientific, USA) at room temperature for 2 hours. Finally, Peroxidase activity was visualized using the ECL Western Blot Detection kit (Beyotime, China). Western blots were quantitated with image analysis system (BioRad, USA). After normalization with $\beta$-actin, data was presented as mean percentages of the ratio of total protein to their respective signal intensity levels found in sham group animals, indicated as $100 \%$.

\section{Real-Time RT-PCR}

Total RNA was extracted from the L4-5 ipsilateral quadrant spinal cord with Trizol and reversed transcribed with the High Capacity cDNA Reverse Transcription Kit (Applied Biosystems, Carlsbad, CA). Real-time PCR was performed using Power SYBR Green Master Mix (Applied Biosystems, Carlsbad, CA) according to the manufacturer's instructions and analyzed by RT-PCR in a detection system (Applied Biosystems, Foster City, CA). The following real-time PCR protocol was followed: reverse transcriptase was activated and CDNA was synthesized $\left(50^{\circ} \mathrm{C}\right.$ for 5 minutes), PCR was activated (95区 for 3 minutes), 40 cycle $s$ of denaturation were performed ( $95^{\circ} \mathrm{C}$ for 30 seconds), and annealing and extension was done for 1 minute at $60^{\circ} \mathrm{C}$. At the end of PCR, a melting curve analysis was performed by slowly increasing the temperature from 60 to $95^{\circ} \mathrm{C}$. The data were analyzed by Software 2.2 using cycle threshold $(\mathrm{Ct})$ value as the readout and relatively normal levels of $\beta$-actin.

The primers used are presented as follows:

- IRF8: 5'-CAAGGGTGTGTTCGTGAAGC-3' (forward), 5'-TCAAAGACCTGCACCACCTC-3'(reverse ).

- Iba1: 5'-GCAAGGATTTGCAGGGAGGA-3'(forward), 5'-TGGGATCATCGAGGAAGTGC-3'(reverse).
- p-P38: 5'-CAGTCCTATCCACGCACCTC-3'(forward), 5'-TGTCGGTGATGTCAGATGGC-3'(reverse).

- $\quad \beta$-actin: 5'-ACTCTGTGTGGATTGGTGGC-3'(forward), 5'-AGAAAGGGTGTAAAACGCAGC-3'(reverse).

\section{Immunofluorescent Histochemistry}

Rats were perfused with $200 \mathrm{~mL}$ saline followed by $200 \mathrm{~mL}$ of $0.1 \mathrm{M}$ phosphate buffer (PB, pH7.3) containing $4 \%$ paraformaldehyde. The L4-5 spinal cord was removed and post-fixed in $4 \%$ paraformaldehyde for 24 hours, and then allowed to equilibrate in 30\% sucrose in PBS overnight at $4^{\circ} \mathrm{C}$. Transverse spinal sections were cryostat cut into 4-6 $\mu \mathrm{m}$, collected in 0.01 M phosphate-buffered saline (PBS, PH7.3). After washing with PBS, the tissue was penetrated with $0.3 \%$ Tritonx-100 at $37^{\circ} \mathrm{C}$ for 20 minutes and blocked with $5 \%$ bovine serum albumin for 1 hour at $37^{\circ} \mathrm{C}$ incubated for 48 hours at $4^{\circ} \mathrm{C}$ with primary antibodies for IRF8/Iba1/pp38 (rabbit anti-IRF8, ab28696, 1:100, Abcam, USA; rabbit anti-lba1, ab178680, 1:100, Abcam, USA; rabbit anti-phosphorylated-p38MAPK (Thr180/Tyr182), \#4631, 1:100, Cell Signaling Technology, USA). The slides were then covered with secondary antibodies containing $1 \mu \mathrm{M}$ DAPI (Sigma). The fluorescence in the spinal cord was monitored with a fluorescent microscope.

\section{Statistical analysis}

All data were analyzed using SPSS 20.0 statistical software package (SPSS Inc., Chicago, USA) and have been presented as mean \pm SEM. All data were graphed using Prism 5.0 (GraphPad, La Jolla, CA). After the data distribution was tested to be normal, behavioral data, western blot data, and ELISA data were analyzed using repeat measures (multiple groups $\times$ time) ANOVA with Bonferroni post hoc test for multiple comparisons to determine overall significance. When ANOVA showed a significant difference, pairwise comparisons between the means were tested by the post-hoc Tukey method or Fisher protected Least Significant Difference (LSD) post-hoc test. An alpha value of 0.05 was considered statistically significant.

\section{Results}

\section{Therapeutic Effects of IRF8 Antisense Oligodeoxynucleotide on Neuropathic Pain}

To investigate the impact of microglial IRF8 in modulating pain, a model in which antisense oligodeoxynucleotide of IRF8 were transferred spinally to rats. It was found $\mathrm{CCl}$-induced allodynia were reversed by in- 

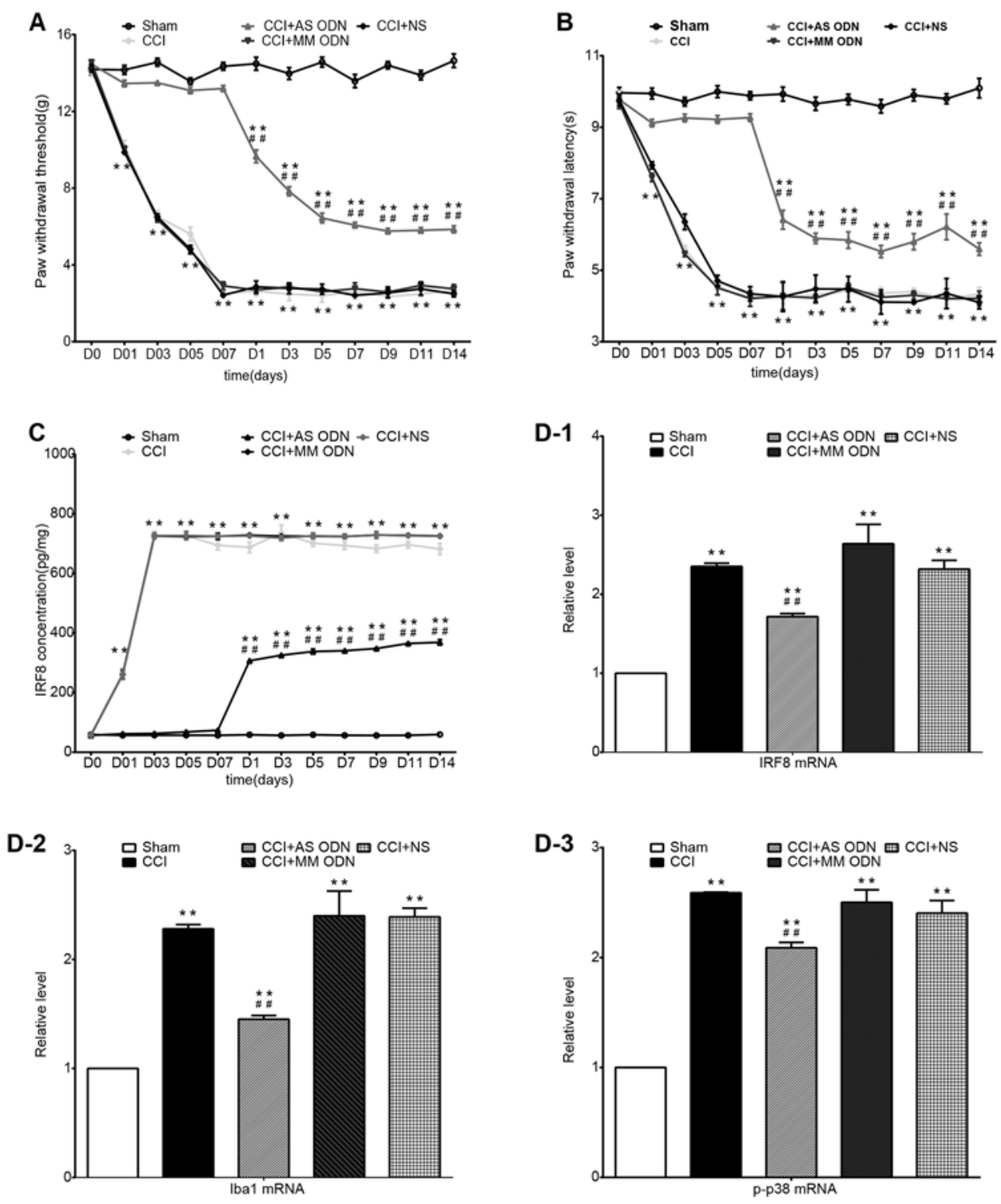

Fig. 1. Therapeutic effects of IRF8 antisense oligodeoxynucleotide on NP. (A-B) Effects of IRF8 oligodeoxynucleotide on mechanical allodynia and thermal hyperalgesia. Reversal of CCI-induced allodynia by intrathecal administration of IRF8 AS ODN once a day for 7 days (12 hours after nerve injury) in rats. Each symbol represents mean $\pm S E M,{ }^{* *} P<0.01$ against Sham group, \#\#P<0.01 against CCI group. Repeat measures (multiple groups $\times$ time) ANOVA, $n=51$ per group. (C) Elisa analysis of IRF8 level in the spinal cord. Each symbol represents mean $\pm S E M,{ }^{* *} P<0.01$ against Sham group, \#\# $<0.01$ against CCI group. LSD-t test, $n=3$ per timepoint per group. (D1-3) Real-time PCR analysis of IRF8, Ibal, p-p38 mRNA in the spinal cord among different groups on D14. Values represent the relative ratio of $I R F 8, I b a 1, p-p 38 \mathrm{mRNA}$ (normalized to $G A P D H \mathrm{mRNA}$ ) to the naive mice. Each symbol represents mean $\pm S E M,{ }^{* *} P<0.01$ against Sham group, \#\#P<0.01 against CCI group. LSD-t test, $n=5$ per group.

trathecal administration of IRF8 AS ODN once a day for 7 days (beginning 12 hours after nerve injury) in rats ( $P$ $<0.01$, Fig. $1 \mathrm{~A}-\mathrm{B})$. Long-lasting mechanical allodynia $(P$ $<0.01$, Fig. 1A) and thermal hyperalgesia $(P<0.01$, Fig.
1B) in $\mathrm{CCl}, \mathrm{CCl}+\mathrm{MM}$ ODN, and $\mathrm{CCl}+\mathrm{NS}$ groups reached a peak on 7th day after nerve injury and maintained stable withdrawal thresholds until the end of observation compared with those in sham-operated rats. The 
E-1
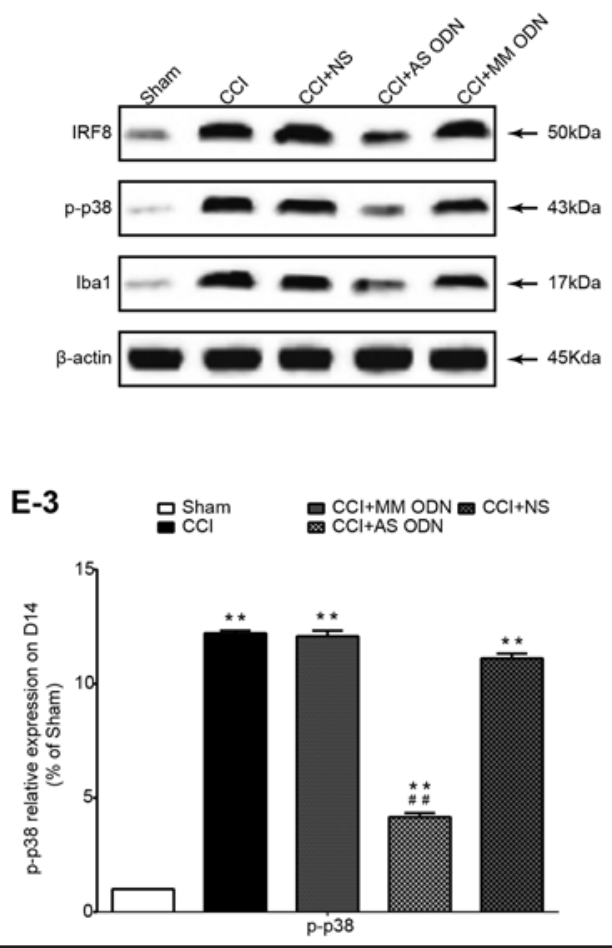
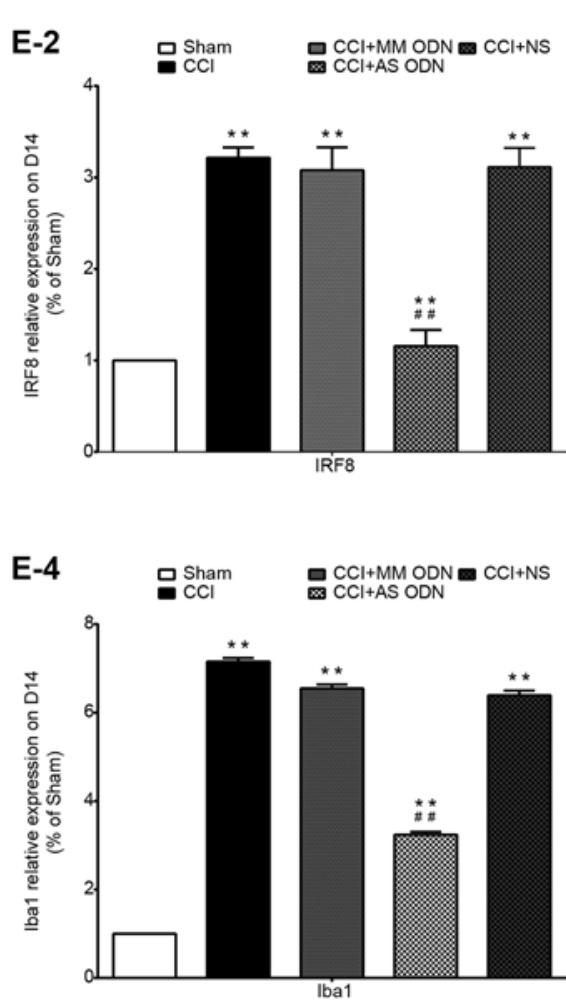

Fig. 1. Therapeutic effects of IRF8 antisense oligodeoxynucleotide on NP. (E1-4) The western blot of IRF8, p-p38, and Ibal proteins in rats' spinal cords among different groups on D14. Values represent the relative ratio of IRF8, Ibal or p-p38 levels (normalized to $\beta$-actin) to the naive mice. Each symbol represents mean $\pm S E M,{ }^{* *} P<0.01$ against Sham group, ${ }^{\# \#} P<0.01$ against CCI. LSD-t test, $n=5$ per assay. (

mechanical allodynia and thermal hyperalgesia were not completely induced and there was no induction of pain behavior during intrathecal injection of IRF8 AS ODN compared with those in Sham group. Although the withdrawal thresholds were significantly decreased after finishing injection in the $\mathrm{CCl}+\mathrm{AS}$ ODN group compared with those in Sham group $(P<0.01)$, but they were still higher than the values in the $\mathrm{CCl}, \mathrm{CCl}+\mathrm{MM}$ ODN and $\mathrm{CCl}+\mathrm{NS}$ groups $(P<0.01)$.

The baseline level of IRF8 was $58.38 \mathrm{pg} / \mathrm{mg}$ as measured by the ELISA analysis. The upregulated IRF8 level started from postoperative day 1, peaked at day 3 , and persisted for at least 21 days in the $\mathrm{CCl}, \mathrm{CCl}+\mathrm{MM}$ $\mathrm{ODN}$ and $\mathrm{CCl}+\mathrm{NS}$ groups compared with those in shamoperated rats $(P<0.01$, Fig. 1C). The levels of IRF8 were stable at low baseline level (56.7-73.7 pg/mg) during intrathecal injection of IRF8 AS ODN, it was increased after finishing injection in $\mathrm{CCl}+\mathrm{AS}$ ODN group compared with those in Sham group $(P<0.01)$, but they were still lower than the values in the $\mathrm{CCl}, \mathrm{CCl}+\mathrm{MM}$ ODN and $\mathrm{CCl}+\mathrm{NS}$ groups $(P<0.01)$.
Correspondingly, RT-PCR analysis (Fig. 1D), western blot (Fig. 1E) and immunofluorescent histochemistry analysis (Fig. 1F) demonstrated that IRF8, Iba1 and p-p38 levels were increased in the ipsilateral spinal cord of the $\mathrm{CCl}, \mathrm{CCl}+\mathrm{MM}$ ODN and $\mathrm{CCl}+\mathrm{NS}$ groups compared with those in Sham group $(P<0.01)$ and decreased in the $\mathrm{CCl}+\mathrm{AS}$ ODN group compared with those in $\mathrm{CCl}$ group $(P<0.01)$ on D14.

\section{Therapeutic Effects of PRF on Neuropathic Pain}

In our study the partial ligation of sciatic nerve induced long-lasting mechanical allodynia $(P<0.01$, Fig. 2A) and thermal hyperalgesia $(P<0.01$, Fig. 2B) after $\mathrm{CCl}$, reached a peak on 7th day, and maintained stable withdrawal thresholds until the end of observation as compared with those in sham-operated rats. No significant changes were found in the contralateral hind paw in all the groups $(P>0.05$, Fig. $2 C-D)$, similar to the ipsilateral paw in Sham group $(P>0.05$, Fig. 2A-B). Mechanical allodynia $(P<0.01$, Fig. $2 A)$ and thermal 
F-1
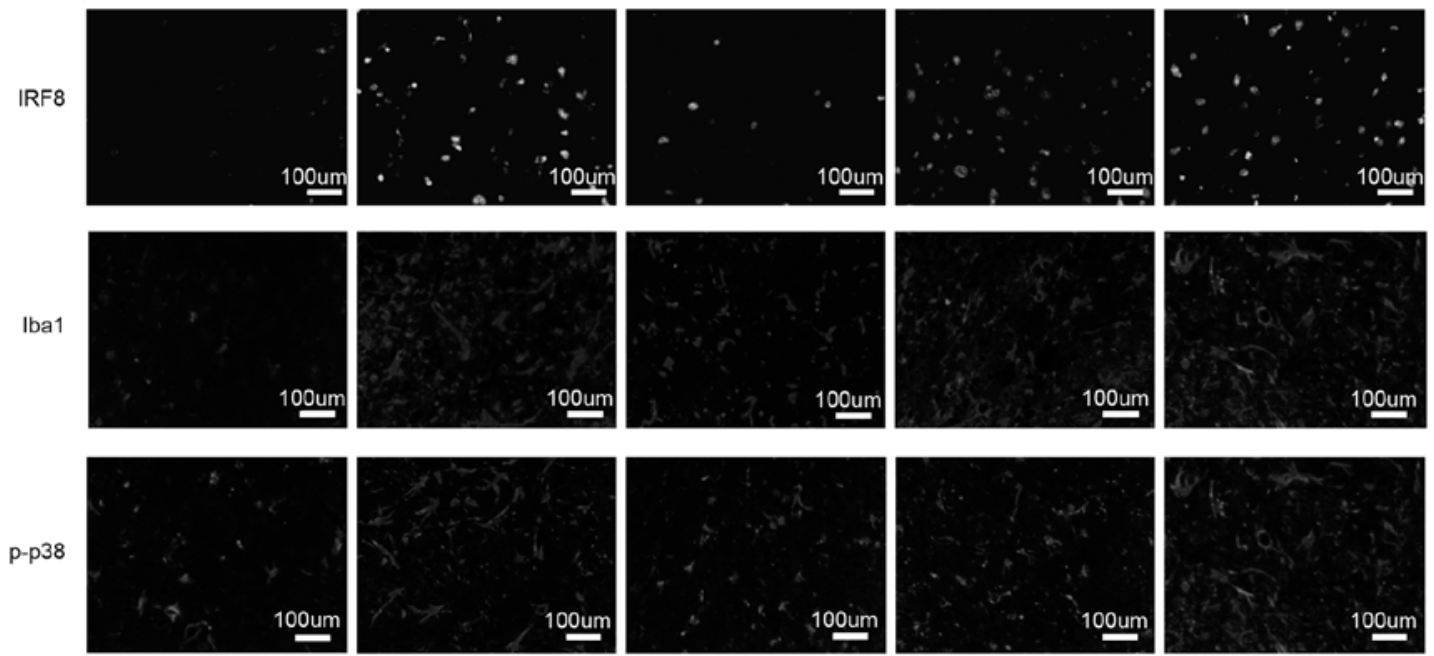

CCI+MM ODN

$\mathrm{CCl}+\mathrm{NS}$

$\mathrm{F}-2$

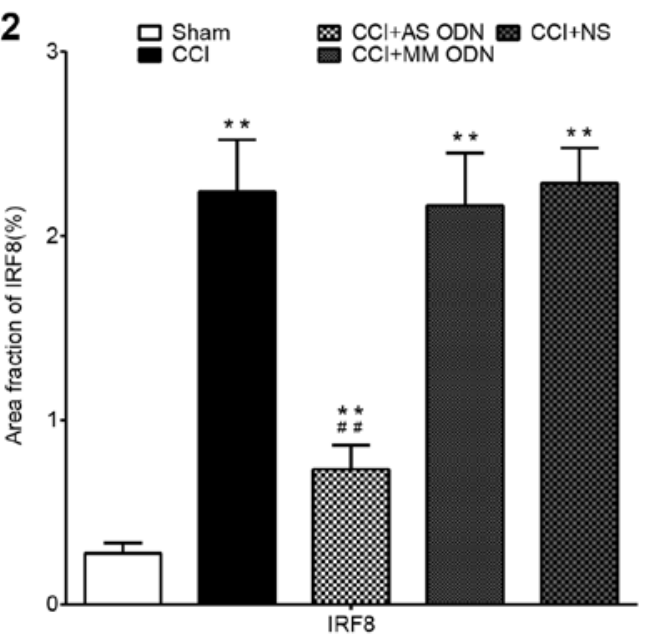

$\mathrm{F}-4$

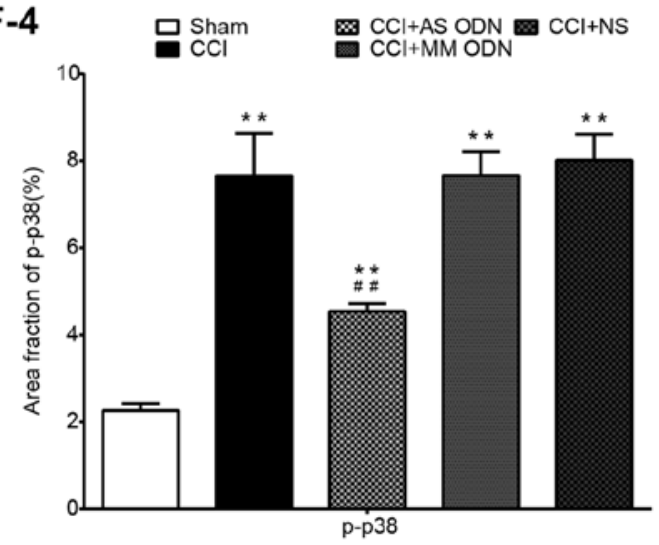

F-3

모군 CCl

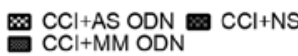

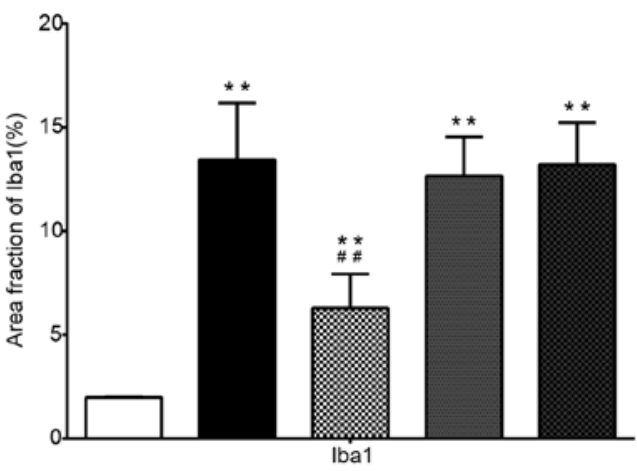

Fig. 1. Therapeutic effects of IRF8 antisense oligodeoxynucleotide on NP. (F1-4) The intensity of IRF8, Ibal, p-p38 immunofluorescence in the spinal cord among different groups on D14. Each symbol represents mean $\pm S E M,{ }^{* *} P<0.01$ against Sham group, ${ }^{\#} P<0.01$ against CCI group. LSD-t test, $n=5$ per group. Scale bars: $100 \mu \mathrm{m}$. 

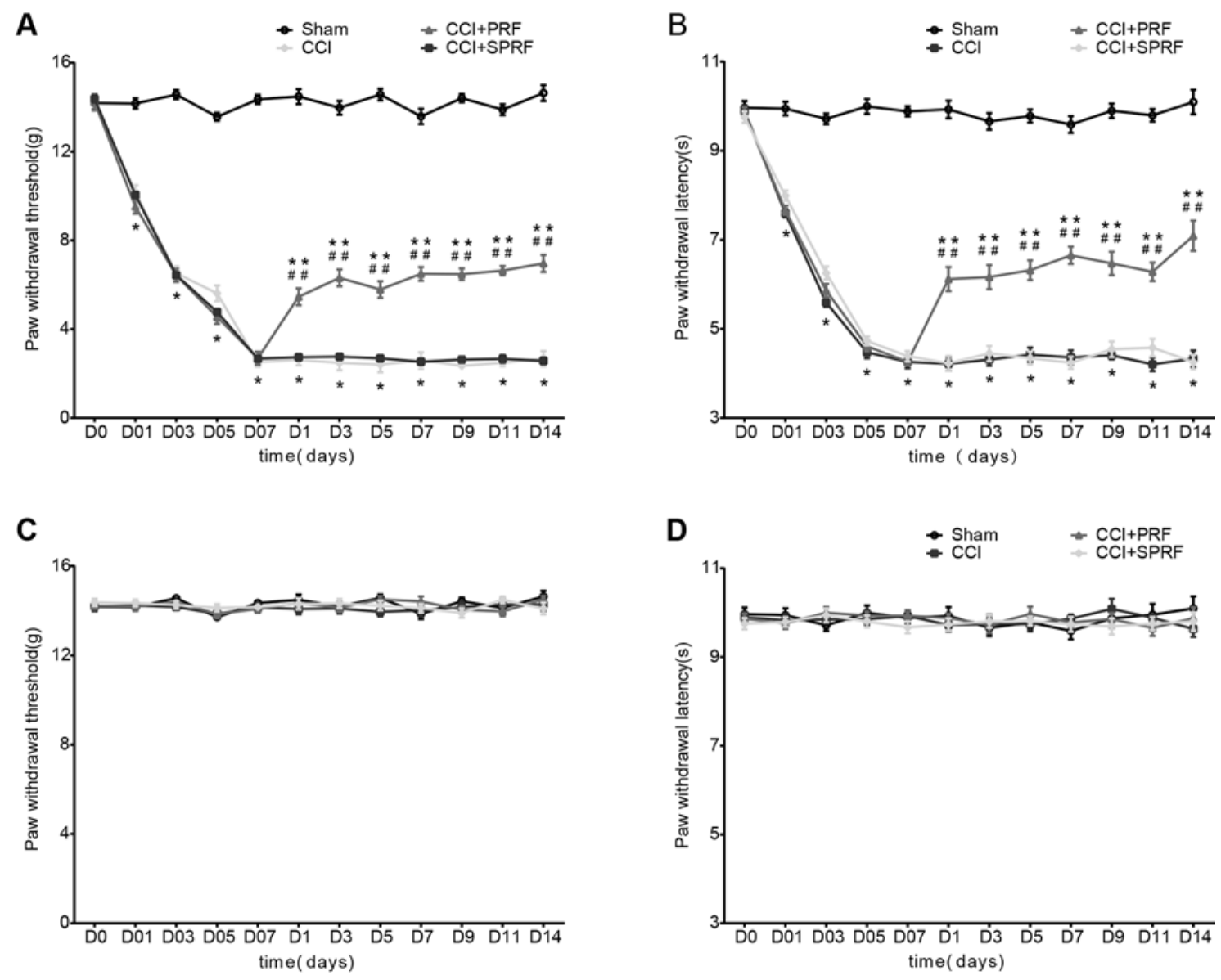

Fig. 2. Therapeutic effects of PRF on neuropathic pain.

CCI induced long-lasting mechanical allodynia $(A)$ and thermal hyperalgesia $(B)$ in ipsilateral hind paw. Changes in paw withdrawal threshold $(C)$ and paw withdrawal latency $(D)$ in contralateral hind paw of all groups. Data is represented as

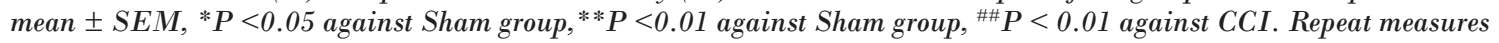
(multiple groups $\times$ time) ANOVA, $n=51$ per group.

hyperalgesia $(P<0.01$, Fig. $2 \mathrm{~B})$ were partial recovered in the $\mathrm{CCl}+\mathrm{PRF}$ group from the first day after a single application of PRF and was maintained throughout a period of 14 days, compared with those in $\mathrm{CCl}$ group, but could not return to pre-CCl baseline. In $\mathrm{CCl}$ and $\mathrm{CCl}$ + SPRF groups, the paw withdrawal threshold and paw withdrawal latency were both maintained low-levels from post lesion days 7 to 21 .

\section{Effects of PRF on the Spinal IRF8, Microglia and p38 Hyperactivity}

The upregulated IRF8 levels decreased from 699.12 $\mathrm{pg} / \mathrm{mg}$ to $381.80 \mathrm{pg} / \mathrm{mg}$ on first day after a single application of PRF $(P<0.01$, against the $\mathrm{CCl}$ group, Fig.
3A) and the levels were stable at $350 \mathrm{pg} / \mathrm{mg}$ for approximately 14 days, although it was still higher than those in Sham group $(P<0.01$, Fig. 3A). Correspondingly, RT-PCR analysis (Fig. 3B), western blot analysis (Fig. 3C) and immunofluorescent histochemistry analysis (Fig. 2D) demonstrated that IRF8 levels increased in the ipsilateral spinal cord of the $\mathrm{CCl}$ group compared with those in Sham group $(P<0.01)$ and decreased in the $\mathrm{CCl}+\mathrm{PRF}$ group compared with those in the $\mathrm{CCl}$ group $(P<0.01)$ on day 14 after a single application of PRF. To understand whether PRF applied to the DRG of rats with $\mathrm{CCl}$ could restrain microglia and p38 hyperactivity in the spinal cord to alleviate neuropathic pain, we performed an RT-PCR analysis (Fig. 3B), a 

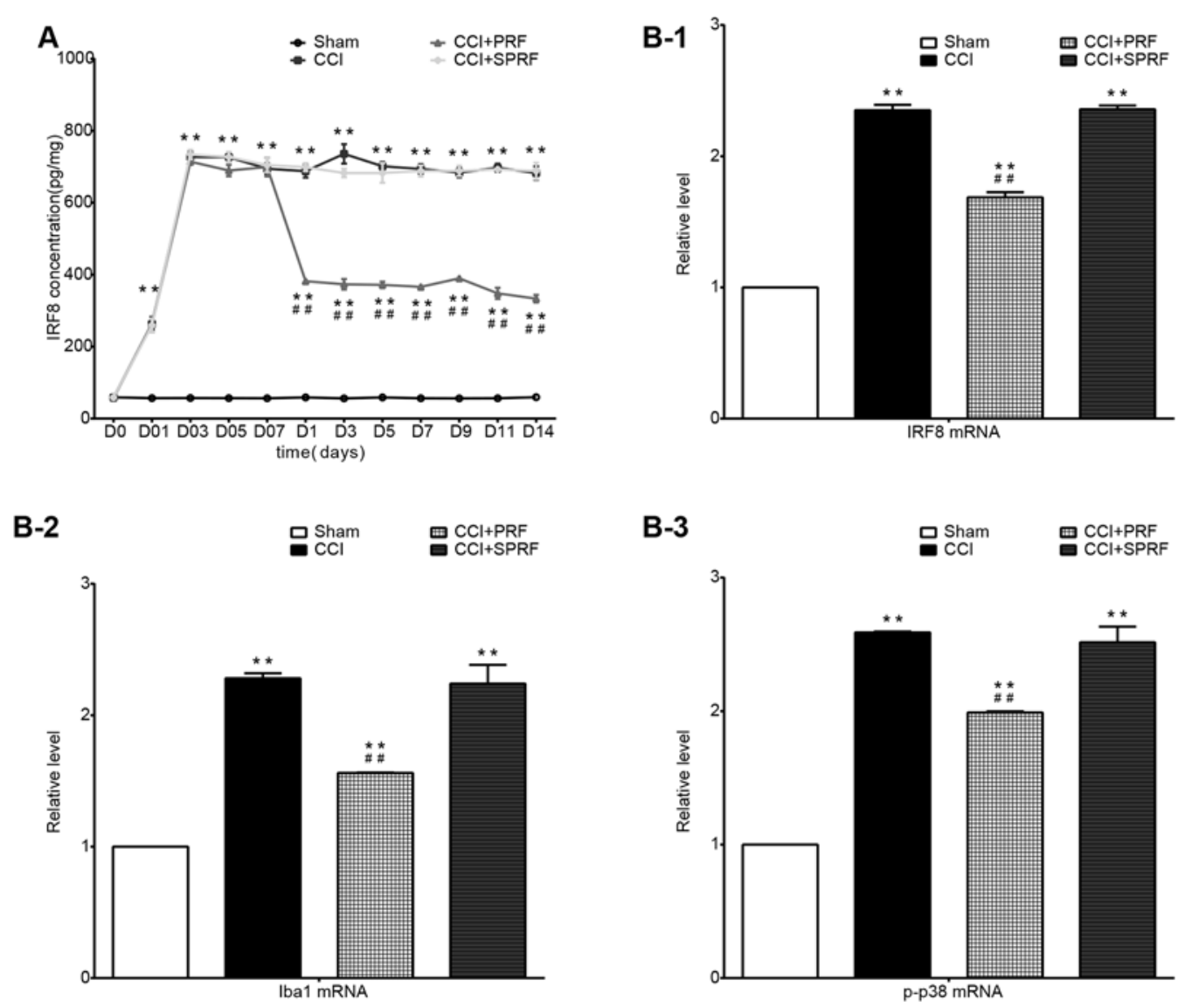

Fig. 3. Effects of PRF on the spinal IRF8, microglia and p38 hyperactivity.

(A) Elisa analysis of IRF8 level in the spinal cord. Each symbol represents mean $\pm S E M, * * P<0.01$ against Sham group, ${ }^{\#} P<0.01$ against CCI group. LSD-t test, $n=3$ per timepoint per group. (B1-3) Real-time PCR analysis of IRF8, Ibal, p-p38 mRNA in the spinal cord among different groups on D14. Values represent the relative ratio of IRF8, Ibal, p-p38 $m R N A$ (normalized to GAPDH $m R N A$ ) to the naive mice. Each symbol represents mean $\pm S E M,{ }^{* *} P<0.01$ against Sham group, ${ }^{\#} P<0.01$ against CCI group. LSD-t test, $n=5$ per group.

western blot analysis (Fig. 3C), and an immunofluorescent histochemistry analysis (Fig. 3D). We found that the expressions of Iba1 $(P<0.01)$ and p-p38 $(P<0.01)$ were significantly increased in the $\mathrm{CCl}, \mathrm{CCl}+\mathrm{SPRF}$, and $\mathrm{CCl}+$ PRF groups compared with those in Sham group after nerve injury. Although after PRF treatment, the expressions of Iba1 $(P<0.01)$ and p-p38 $(P<0.01)$ in the $\mathrm{CCl}+\mathrm{PRF}$ group decreased significantly than those in the $\mathrm{CCl}, \mathrm{CCl}+$ SPRF groups, ut were still higher than those in the Sham group $(P<0.01)$.

\section{PRF Therapy Took No Effect in Normal Rats}

We further examined if PRF could alter pain behaviors and pain signals in normal rats. All PRF-treated rats without $\mathrm{CCl}$ exhibited normal movements. The mechanical allodynia and thermal hyperalgesia were not found, and there were no differences in the ipsilateral paws between Sham + PRF and Sham groups at all time points $(P>0.05$, Fig.4 A-B). Similarly, no differences were found in the expressions of IRF8, Iba1, and p-p38 between Sham and Sham + PRF groups $(P>0.05$, Fig. $4 C-F)$.

\section{Discussion}

\section{Effects of PRF Therapy on Pain Behaviors}

\section{Behavioral Changes Induced by PRF on the DRG of CCI Rats}

DRG is an oval inflation of the dorsal root at the upper region of the intervertebral foramen, which contains the first class of neurons of sensory afferents. The 
C-1
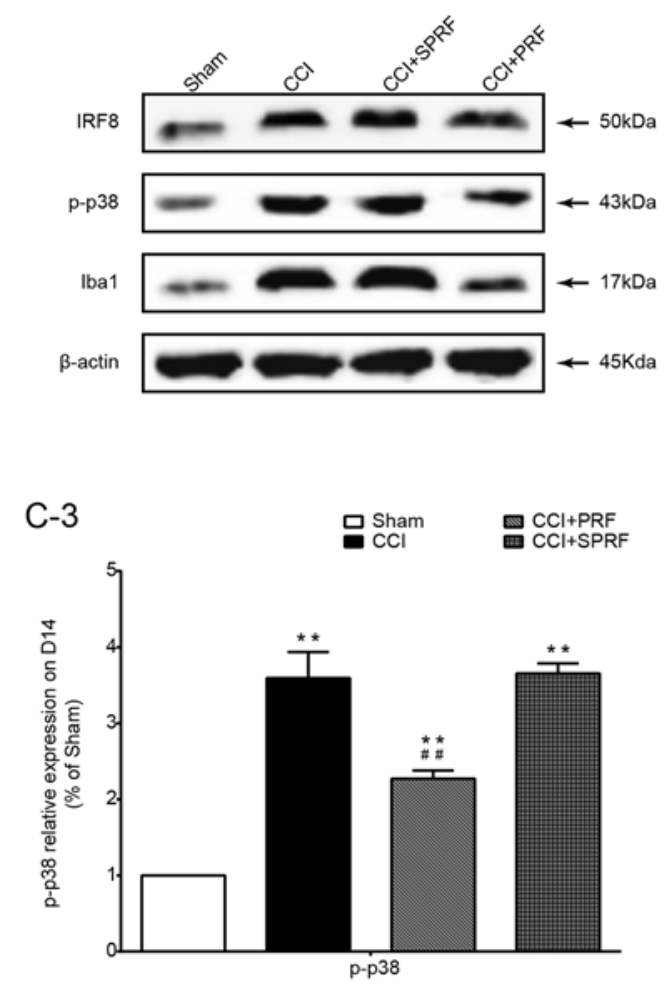

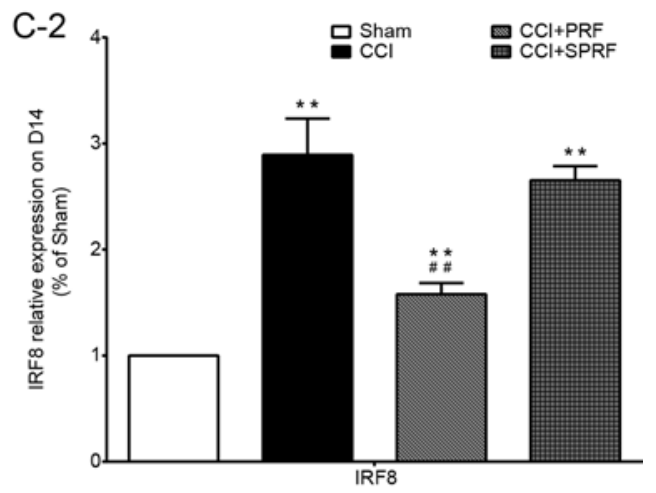

C-4

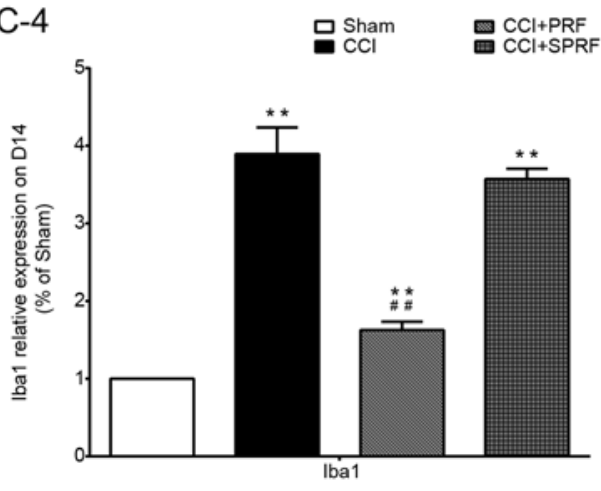

Fig. 3. Effects of PRF on the spinal IRF8, microglia and p38 hyperactivity.

(C1-4) The western blot of IRF8, p-p38, and Ibal proteins in rats spinal cords among different groups on D14. Values represent the relative ratio of IRF8, Ibal or p-p38 levels (normalized to $\beta$-actin) to the naive mice. Each symbol represents mean $\pm S E M,{ }^{* *} P<0.01$ against Sham group, ${ }^{\# \#} P<0.01$ against CCI. LSD-t test, $n=5$ per assay.

activation of immune and immune-like glial cells in the DRG and spinal cord leads to the release of both proand anti-inflammatory cytokines, which are involved in the spinal nociceptive transmission and central sensitization $(21,22)$. Due to its important role in the modulation of sensory processing and its anatomic accessibility to clinical intervention, it becomes an excellent clinical target for pain treatment (23-26). A previous study showed that the increased exposure time to PRF current from 2 to 6 minutes contributed to an enhanced anti-allodynic effect without motor impairment (27). Thus, in our study, the PRF current duration was set for 6 minutes to the L4-5 DRG to gain pain relief.

In this study, we developed the rat's $\mathrm{CCl}$ model according to a previous report to mimic the neuropathic pain manifestations in human (17). Mechanical allodynia and thermal hyperalgesia were partial recovered from 1 day after a single application of PRF and maintained throughout a period of 14 days. The reasons which mechanical allodynia and thermal hyperalgesia were reversed even after PRF cessation but did not recover to pre-CCl baseline, maybe correlated to the conditions, such as voltage of PRF (45V), duration (6 $\mathrm{min})$, and $\mathrm{CCl}$ model. It remains to be investigated if the upregulated voltage (over 45V) and prolonged duration (over $6 \mathrm{~min}$ ) are capable of achieving more beneficial pain relief.

\section{Prolonged Analgesia of PRF}

The activation of mitogen-activated protein kinases (MAPKs) which contain p38, extracellular regulated protein kinase (ERK) and c-Jun N-terminal kinase (JNK) induces synthesis of pro-inflammatory/pronociceptive 


\section{D-1}

IRF8
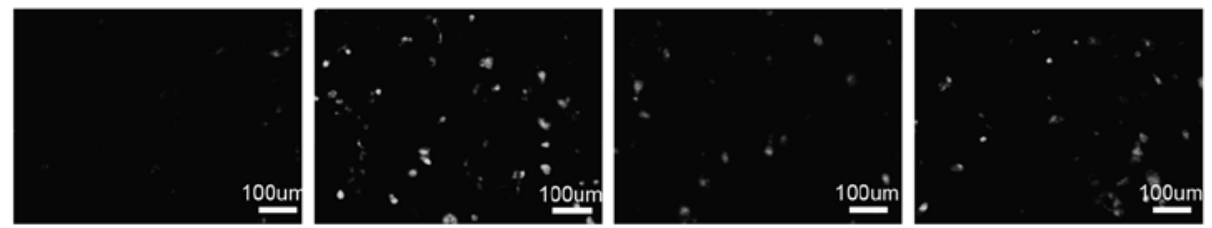

lba1
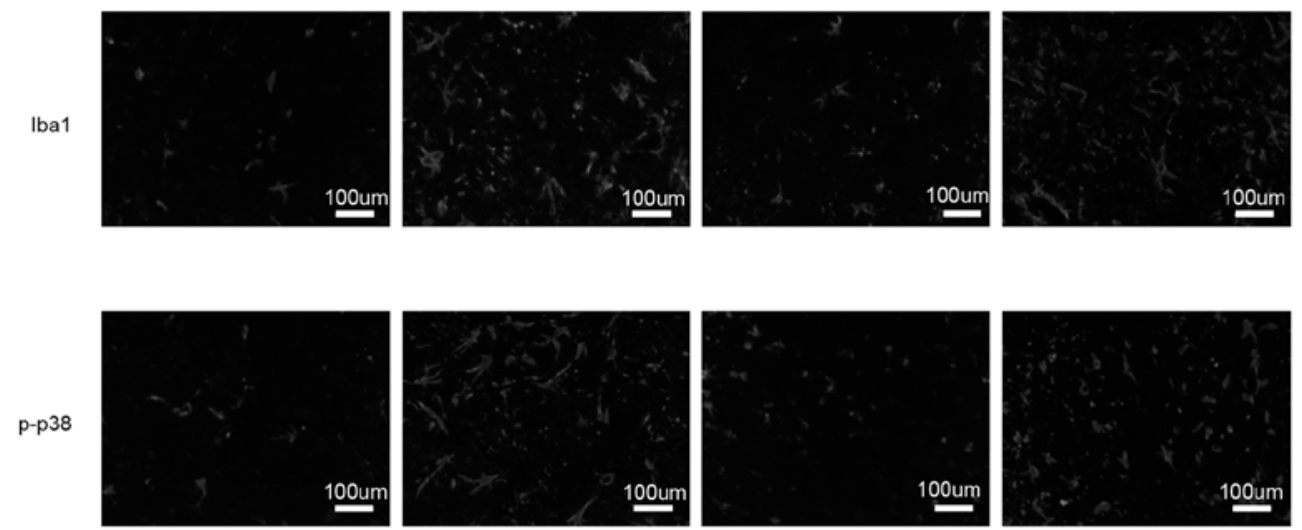

Sham

$\mathrm{CCl}$

PRF

SPRF

D-2

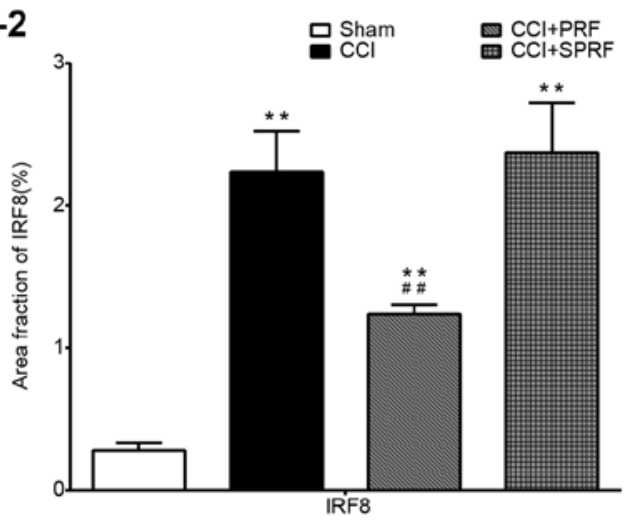

D-4

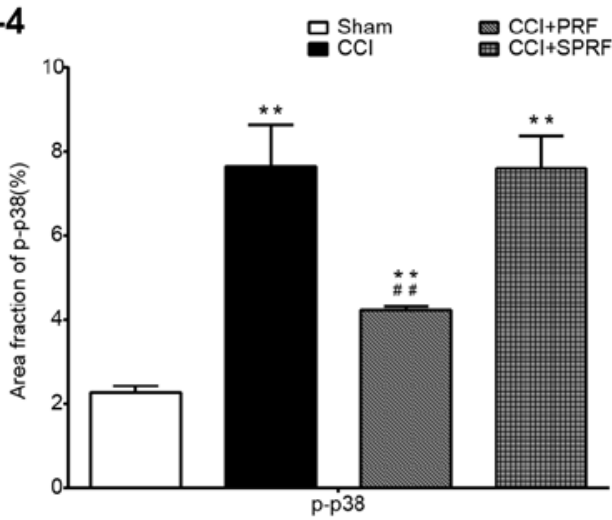

D-3

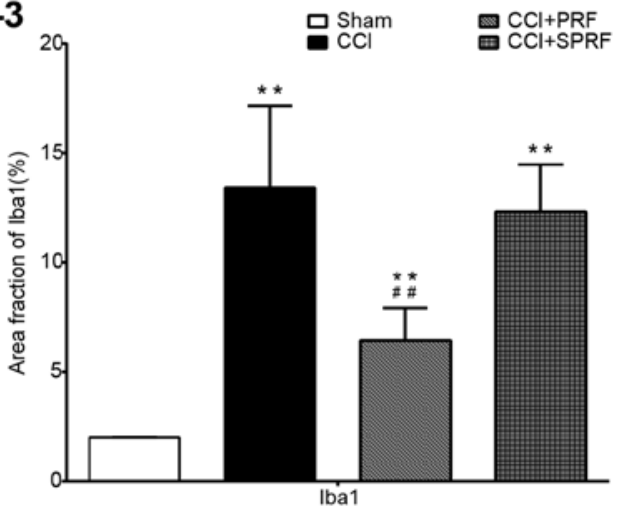

Fig. 3. Effects of PRF on the spinal IRF8, microglia and p38 hyperactivity.

(D1-4) The intensity of IRF8, Ibal, p-p38

immunofluorescence in the spinal cord among different groups on D14. Each symbol represents mean $\pm S E M$, ${ }^{* *} P<0.01$ against sham group, ${ }^{\# \#} \boldsymbol{P}<0.01$ against $C C I$ group. LSD-t test, $n=5$ per group. Scale bars: $100 \mu \mathrm{m}$. 

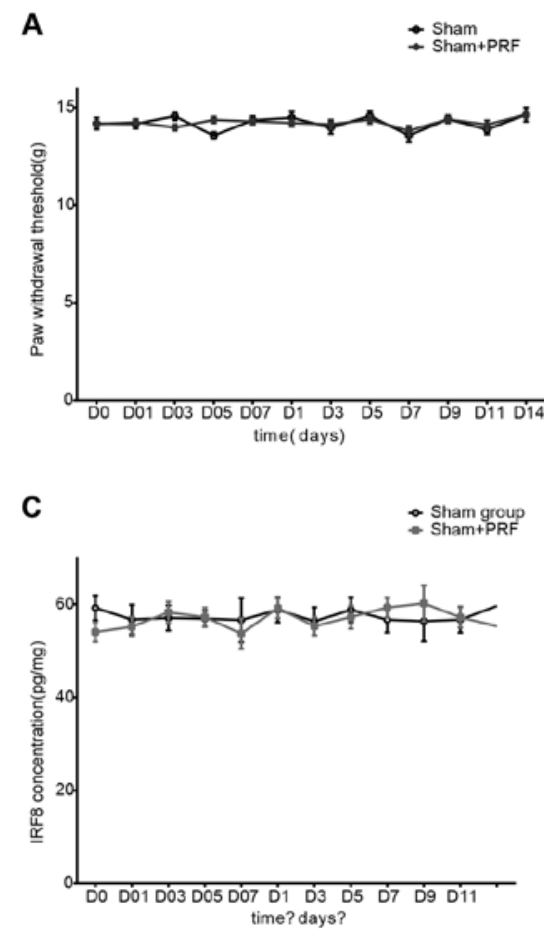

E-1

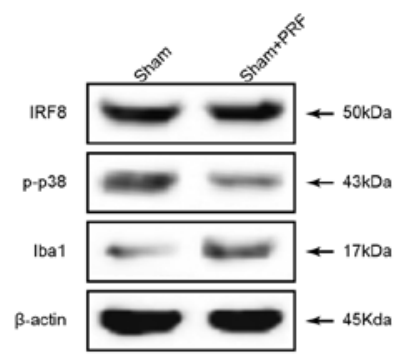

F-1

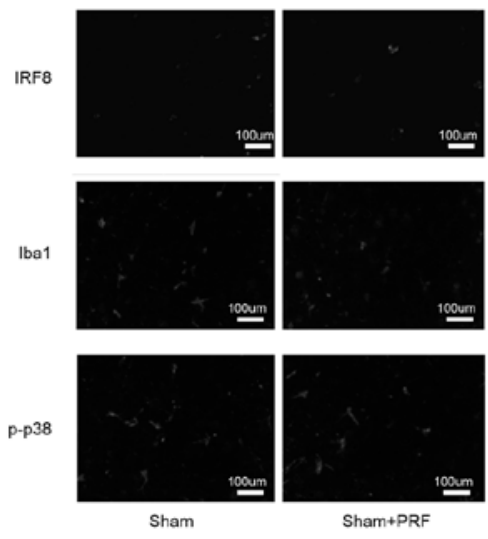

B

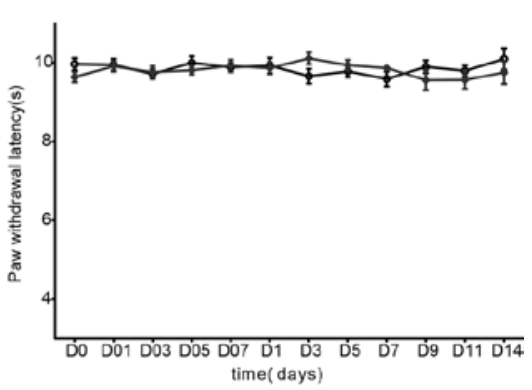

D

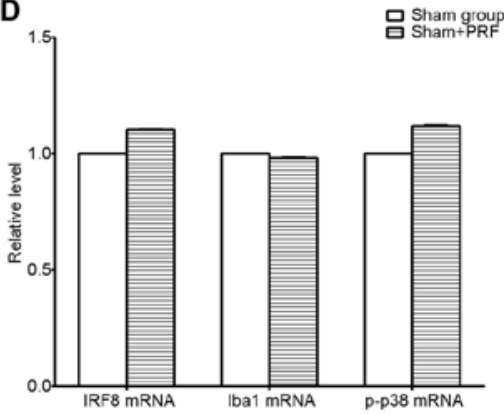

E-2

믐 Sham group
Sham+PRF

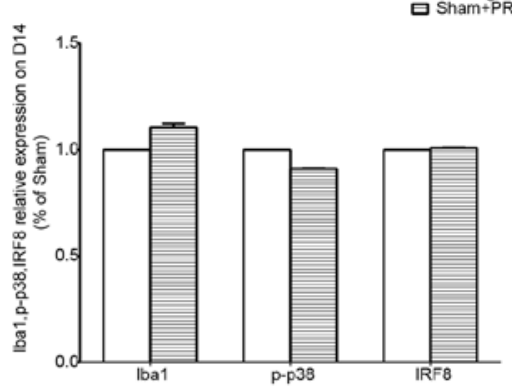

F-2

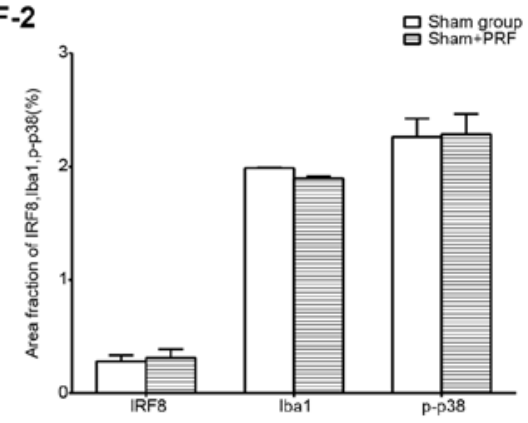

Fig. 4. PRF therapy induced no effect in healthy tissue. (A-B) Effect of PRF on mechanical allodynia and thermal hyperalgesia applied to L4-5 DRG in normal rats. Each symbol represents mean \pm SEM. Repeat measures (multiple groups $\times$ time) ANOVA, $n=51$ per group. (C) Elisa analysis of IRF8 level in the spinal cord. Each symbol represents mean \pm SEM. LSD-t test, $n=3$ per timepoint per group. (D) Real-time PCR analysis of IRF8, Ibal, p-p38 mRNA in the spinal cord among different groups on D14. Values represent the relative ratio of IRF8, Ibal, p-p38 $m R N A$ (normalized to GAPDH mRNA) to the naive mice. Each symbol represents mean $\pm S E M$. $L S D$-t test, $n=5$ per group. (E1-2) The western blot of IRF8, p-p38, and IbaI proteins in rats' spinal cords among different groups on D14. Values represent the relative ratio of $I R F 8, I b a I$ or p-p38 levels (normalized to $\beta$-actin) to the naive mice. Each symbol represents mean $\pm S E M$. LSD-t test, $n=5$ per assay. (F1-2) The intensity of IRF8, Ibal, p-p38 immunofluorescence in the spinal cord among different groups on D14. Each symbol represents mean $\pm S E M$. LSD-t test, $n=5$ per group. Scale bars: $100 \mu \mathrm{m}$. 
mediators to enhance and prolong neuropathic pain (7). Early application of PRF adjacent to the DRG can downregulate p38 and ERK activation, along with alleviating nerve ligation-induced mechanical allodynia for 7 days and thermal hyperalgesia on postoperative days 3-7 (28). It has been previously demonstrated that PRF inhibits SNL-induced microglial p-p38 and TNF (28). Also, PRF on the ipsilateral DRG can reverse mechanical allodynia induced by autologous nucleus pulposus implantation to a nerve root for 40 days (8). In our study the benefits of a single PRF application persisted for at least 2 weeks, which further confirmed that PRF on DRG could contribute to a prolonged analgesic efficacy. We also observed that pain reduction was accompanied by the reversed IRF8-increased expression, microglial activation, and p38 phosphorylation in the spinal cord after PRF therapy. Thus, the inhibition of IRF8, microglia, p-p38 might participate in the prolonged analgesia.

Long-term potentiation (LTP), an increase in postsynaptic current or potential evoked by a single monosynaptic preganglionic action potential, is involved in nociceptive sensitizing processes. It sensitizes molecules in the central nervous system to maintain and enhance pathological pain (29-31). Spinal glia sustain late-phase LTP in neuropathic pain for days, weeks, or even longer (32-34). Our study demonstrated that PRF intervention inhibited spinal microglial expression. Moreover, application of bipolar PRF adjacent to the L5 DRG of SNL and control at an intensity of $5 \mathrm{~V}$ for 5 minutes can lead to selective long-term depression (LTD) of PRF on C-fiber-mediated spinal evoked-field potentials (35). Therefore, our further objective is to explore how PRF on DRG of CCI rats alters LTP or LTD to exert prolonged analgesia.

\section{The Mechanisms of Analgesic Effect of PRF}

\section{PRF Effects on Spinal Microglia/p38 Hyperactivity}

Evidence has demonstrated the pivotal role of spinal microglia in neuropathic pain (36). Microglia cells are the first to be activated following various insults in the nervous system, including PNI and they remain active for several weeks $(21,37)$. Microglia transform to reactive phenotype and display a progressive series of cellular and molecular changes, including morphological hypertrophy, rapid proliferation, upregulated expression of various genes, and increased expression of microglia characteristic markers, such as Iba1, and increased p38 phosphorylation in the spinal microglia (15,38-42). The inflammatory cytokines are then released by microglia in a p38-dependent way and contribute to the development of pain hypersensitization and long-persisting pain (43). It is noteworthy that p38 is activated only in the spinal microglia after nerve injury (18). Early studies showed that minocycline and SB203580 could prevent/delay neuropathic pain (44).

PRF was applied to the DRG in a rat model of neuropathic pain and observed the reduction of established mechanical hypersensitivity and significant attenuation of activation of microglia in the spinal dorsal horn $(7,8)$. Our data is consistent with conclusions from previous studies as mentioned before. The mechanical allodynia and thermal hyperalgesia were recovered, the expression of Iba1 and p-p38 were significantly downregulated after a single PRF, although it didn't recover to normal value. This demonstrated that PRF may suppress the activation of microglia and p38 signaling pathway and contributed to the nociceptive relief.

\section{PRF Effects on Spinal IRF8}

Microglial cells are activated and converted to reactive phenotype after PNI. But, we also wanted to examine how microglia transformed into reactive phenotypes. IRF8 plays a crucial role for microglial activation and is involved in the development of neuropathic pain (16). It has been shown that IRF8 expression significantly increased only in the spinal microglia after PNI, and IRF8-knockout mice showed resistance to PNI-induced tactile allodynia. IRF8 small interfering RNA was intrathecal injected on day 5 and 6 into post-PNI wild type mice that had established neuropathic pain, and there was a significant recovery in PWT. In vitro studies also suggest that IRF8 overexpression in microglia activates the transcription of genes associated with the microglial reactive states, including toll-like receptor 2 , chemotaxis, and inflammatory components. These genes were inhibited in IRF8-knockout mice spinal cord following PNI. Thus, IRF8 regulates a series of gene expressions that transform microglia into a reactive phenotype and initiates neuropathic pain. In addition, there is a reversal of $\mathrm{CCl}$-induced allodynia and presence of high levels of IRF8, Iba1, and p-p38 in the spinal cord after intrathecal administration of IRF8 AS ODN in rats. These results further imply that IRF8 should be located on the upstream of microglia and $\mathrm{p} 38$.

IRF8 is the key for microglial activation, and PRF to the DRG in rats with neuropathic pain could induce pain-relief via lessening microglial activation. So, we deduced that PRF might regulate microglial activation through blocking the expression of IRF8 in the spinal 
cord. We observed that spinal IRF8 levels were significantly and persistently downregulated for 14 days after PRF treatment, consistent with the analgesic effects on mechanical allodynia and thermal hyperalgesia. Moreover, PRF induced long-lasting inhibition of microglial activity and p-p38 in the spinal cord and rescued the sensory function even after interruption of treatment. This reveals that PRF on the DRG mimics IRF8 AS ODN to contribute to the pain reduction. Nerve injury-induced microglial proliferation in the spinal cord is not influenced by IRF8-knockout (16); therefore, it is not due to defect in reactive processes of microglia due to lack of IRF8. The results could explain the fact that IRF8 and microglia were significantly downregulated but the values still higher than the normal baseline after PRF treatment for an established chronic pain syndrome.

\section{PRF: A Safe Therapy in Pain Management}

PRF treatment offers several unique advantages, including minimal invasiveness, ease of application, and greater safety due to the lower heat emission which enormously reduces the risk of nerve damage (35). Serious adverse effects, including infection, long-term neurological deficits, nerve injury, postoperative paresthesia, dysesthesias, or hyposensitivity following the correct PRF treatment were rarely reported (5,45-47). Choi et al (48) applied $42^{\circ} \mathrm{C}$ PRF directly on the normal sciatic nerve of rats for 2 minutes and only minor injury and edema in the myelinated axons were observed on the second day after procedure. In this study, all PRFtreated rats without $\mathrm{CCl}$ exhibited normal movement and there was no mechanical allodynia and thermal hy- peralgesia. There was no difference in the spinal levels of IRF8, Iba1, and p-p38 between the groups of Sham and Sham + PRF. Thus, we conclude that there are no adverse effects of PRF to the DRG in the spinal cord and thus, is safe for therapy in pain management.

\section{Limitations}

In this study, we did not do additional research to evaluate the most appropriate treatment parameters of PRF for treating neuropathic pain. In vivo field potential recording in PRF-treated rats is absent. Also, we just selected one time-point to assay the levels of IRF8, Iba1, and p-p38. The changes of IRF8, Iba1, p-p38 levels in the ipsilateral DRG were not investigated. More detailed research about PRF on the DRG is needed to ease the neuropathic pain further.

\section{Conclusions}

To our knowledge, this is the first study that employs PRF on the DRG to partially reverse $\mathrm{CCl}$-induced neuropathic pain beyond the treatment period. Additionally, we show that PRF suppresses IRF8 upregulation and microglia, p-p38 hyperactivity in the spinal cord to alleviate neuropathic pain. Our findings could elucidate the proper targets for pain management and provide therapeutic targets to neuropathic pain. It supports PRF treatment as a valuable intervention therapy for chronic neuropathic pain. The treatment will become more optimized for patients due to PRF as a viable clinical option. Future basic science research, and larger, long-term outcome, controlled clinical trials are needed to understand the efficacy and the mechanism of PRF clearly

\section{References}

1. Jensen TS, Baron R, Haanpaa M, Kalso E, Loeser JD, Rice AS, Treede RD. A new definition of neuropathic pain. Pain 2011; 152:2204-2205.

2. Truini A, Garcia-Larrea L, Cruccu G. Reappraising neuropathic pain in humans-how symptoms help disclose mechanisms. Nat Rev Neurol 2013; 9:572-582.

3. Udina E, Cobianchi S, Allodi I, Navarro $X$. Effects of activity-dependent strategies on regeneration and plasticity after peripheral nerve injuries. Ann Anat 2011; 193:347-353.

4. Dahan A, Olofsen E, Niesters M. Pharmacotherapy for pain: Efficacy and safety issues examined by subgroup analy- ses. Pain 2015; 156 Suppl 1: S119-126.

5. Shanthanna H, Chan P, McChesney J, Thabane L, Paul J. Pulsed radiofrequency treatment of the lumbar dorsal root ganglion in patients with chronic lumbar radicular pain: A randomized, placebocontrolled pilot study. J Pain Res 2014; 7:47-55.

6. Nizard J, Lefaucheur JP, Helbert M, de Chauvigny E, Nguyen JP. Non-invasive stimulation therapies for the treatment of refractory pain. Discov Med 2012; 14:21-31.

7. Park HW, Ahn SH, Son JY, Kim SJ, Hwang SJ, Cho YW, Lee DG. Pulsed radiofrequency application reduced me- chanical hypersensitivity and microglial expression in neuropathic pain model. Pain Med 2012; 13: 1227-1234.

8. Cho HK, Cho YW, Kim EH, Sluijter ME, Hwang SJ, Ahn SH. Changes in pain behavior and glial activation in the spinal dorsal horn after pulsed radiofrequency current administration to the dorsal root ganglion in a rat model of lumbar disc herniation: Laboratory investigation. J Neurosurg Spine 2013; 19: 256-263.

9. Glass CK, Saijo K, Winner B, Marchetto $\mathrm{MC}$, Gage $\mathrm{FH}$. Mechanisms underlying inflammation in neurodegeneration. Cell 2010; 140: 918-934.

10. Perry VH Holmes C. Microglial priming 
in neurodegenerative disease. Nat Rev Neurol 2014; 10:217-224.

11. Ransohoff RM Cardona AE. The myeloid cells of the central nervous system parenchyma. Nature 2010; 468:253-262.

12. Rojewska E, Makuch W, Przewlocka B, Mika J. Minocycline prevents dynorphin-induced neurotoxicity during neuropathic pain in rats. Neuropharmacology 2014; 86:301-310.

13. Yasuda S, Sugiura H, Tanaka H, Takigami S, Yamagata K. p38 MAP kinase inhibitors as potential therapeutic drugs for neural diseases. Cent Nerv Syst Agents Med Chem 2011; 11:45-59.

14. Liu N, Zhang D, Zhu M, Luo S, Liu T. Minocycline inhibits hyperpolarizationactivated currents in rat substantia gelatinosa neurons. Neuropharmacology 2015; 95:110-120.

15. Choi DC, Lee JY, Lim EJ, Baik HH, Oh TH, Yune TY. Inhibition of ROS-induced P38MAPK and ERK activation in microglia by acupuncture relieves neuropathic pain after spinal cord injury in rats. Exp Neurol 2012; 236: 268-282.

16. Masuda T, Tsuda M, Yoshinaga R, Tozaki-Saitoh $\mathrm{H}$, Ozato K, Tamura T, Inoue $\mathrm{K}$. IRF8 is a critical transcription factor for transforming microglia into a reactive phenotype. Cell Rep 2012; 1: 334-340.

17. Li DY, Meng L, Ji N, Luo F. Effect of pulsed radiofrequency on rat sciatic nerve chronic constriction injury: A preliminary study. Chin Med J (Engl) 2015; 128: 540-544.

18. Geng SJ, Liao FF, Dang WH, Ding X, Liu XD, Cai J, Han JS, Wan Y, Xing GG. Contribution of the spinal cord BDNF to the development of neuropathic pain by activation of the $\mathrm{NR}_{2} \mathrm{~B}$-containing NMDA receptors in rats with spinal nerve ligation. Exp Neurol 2010; 222:256-266.

19. Chaplan SR, Bach FW, Pogrel JW, Chung JM, Yaksh TL. Quantitative assessment of tactile allodynia in the rat paw. J Neurosci Methods 1994; 53:55-63.

20. Hargreaves K, Dubner R, Brown F, Flores $C$, Joris J. A new and sensitive method for measuring thermal nociception in cutaneous hyperalgesia. Pain 1988; 32:77-88.

21. Ikeda H, Kiritoshi T, Murase K. Contribution of microglia and astrocytes to the central sensitization, inflammatory and neuropathic pain in the juvenile rat. Mol Pain 2012; 8:43.

22. Liem L, van Dongen E, Huygen FJ, Staats $\mathrm{P}$, Kramer J. The dorsal root ganglion as a therapeutic target for chronic pain. Reg Anesth Pain Med 2016; 41:511-519.

23. Kim K, Jo D, Kim E. Pulsed Radiofrequency to the dorsal root ganglion in acute herpes zoster and postherpetic neuralgia. Pain Physician 2017; 20E411-e418.

24. Kanda H Gu JG. Membrane mechanics of primary afferent neurons in the dorsal root ganglia of rats. Biophys ] 2017; 112:1654-1662.

25. Berta T, Qadri Y, Tan PH, Ji RR. Targeting dorsal root ganglia and primary sensory neurons for the treatment of chronic pain. Expert Opin Ther Targets 2017:1-9.

26. Krames ES. The dorsal root ganglion in chronic pain and as a target for neuromodulation: A review. Neuromodulation 2015; 18:24-32; discussion 32.

27. Tanaka N, Yamaga M, Tateyama S, Uno T, Tsuneyoshi I, Takasaki M. The effect of pulsed radiofrequency current on mechanical allodynia induced with resiniferatoxin in rats. Anesth Analg 2010; 111:784-790.

28. Lin ML, Lin WT, Huang RY, Chen TC, Huang $\mathrm{SH}$, Chang $\mathrm{CH}$, Tsai SY, Chiu HW, Yeh GC, Lin CW, Wen YR. Pulsed radiofrequency inhibited activation of spinal mitogen-activated protein kinases and ameliorated early neuropathic pain in rats. Eur ] Pain 2014; 18:659-670.

29. Sandkuhler J Gruber-Schoffnegger D. Hyperalgesia by synaptic long-term potentiation (LTP): An update. Curr Opin Pharmacol 2012;12: 18-27.

30. Pfau DB, Klein T, Putzer D, PogatzkiZahn EM, Treede RD, Magerl W. Analysis of hyperalgesia time courses in humans after painful electrical high-frequency stimulation identifies a possible transition from early to late LTP-like pain plasticity. Pain 2011; 152:1532-1539.

31. Chen T, O’Den G, Song Q, Koga K, Zhang MM, Zhuo M. Adenylyl cyclase subtype 1 is essential for late-phase long term potentiation and spatial propagation of synaptic responses in the anterior cingulate cortex of adult mice. $\mathrm{Mol}$ Pain 2014; 10: 65 .

32. Chu YX, Zhang $Y$, Zhang $Y Q$, Zhao ZQ. Involvement of microglial $\mathrm{P}_{2} \mathrm{X}_{7}$ receptors and downstream signaling pathways in long-term potentiation of spinal nociceptive responses. Brain Behav Immun 2010; 24:1176-1189.

33. Liang L, Wang Z, Lu N, Yang J, Zhang Y, Zhao Z. Involvement of nerve injury and activation of peripheral glial cells in tetanic sciatic stimulation-induced persistent pain in rats. J Neurosci Res 2010; 88:2899-2910.

34. Liu XG Zhou LJ. Long-term potentiation at spinal C-fiber synapses: A target for pathological pain. Curr Pharm Des 2015; 21:895-905.

35. Huang RY, Liao CC, Tsai SY, Yen CT, Lin $\mathrm{CW}$, Chen TC, Lin WT, Chang $\mathrm{CH}$, Wen YR. Rapid and delayed effects of pulsed radiofrequency on neuropathic pain: Electrophysiological, molecular, and behavioral evidence supporting long-term depression. Pain Physician 2017; 20: E269-e283.

36. Tsuda M. Microglia in the spinal cord and neuropathic pain. Journal of Diabetes Investigation 2016; 7: 17-26.

37. Tenorio G, Kulkarni A, Kerr BJ. Resident glial cell activation in response to perispinal inflammation leads to acute changes in nociceptive sensitivity: Implications for the generation of neuropathic pain. Pain 2013; 154: 71-81.

38. Taves S, Berta T, Liu DL, Gan S, Chen G, Kim YH, Van de Ven T, Laufer S, Ji RR. Spinal inhibition of p38 MAP kinase reduces inflammatory and neuropathic pain in male but not female mice: Sexdependent microglial signaling in the spinal cord. Brain Behav Immun 2016; 55: 70-81.

39. Tan YH, Li K, Chen XY, Cao Y, Light AR, Fu KY. Activation of Src family kinases in spinal microglia contributes to formalin-induced persistent pain state through p38 pathway. J Pain 2012; 13:1008-1015.

40. Sun JS, Yang YJ, Zhang YZ, Huang W, Li ZS, Zhang Y. Minocycline attenuates pain by inhibiting spinal microglia activation in diabetic rats. Mol Med Rep 2015; 12:2677-2682.

41. Ni HD, Yao M, Huang B, Xu LS, Zheng Y, Chu YX, Wang HQ, Liu MJ, Xu SJ, Li HB. Glial activation in the periaqueductal gray promotes descending facilitation of neuropathic pain through the p38 MAPK signaling pathway. J Neurosci Res 2016;94: 50-61.

42. Berta T, Qadri YJ, Chen G, Ji RR. Microglial signaling in chronic pain with a special focus on Caspase 6, p38 MAP kinase, and sex dependence. J Dent Res 2016; 95:1124-1131.

43. Mika J, Zychowska M, Popiolek-Barczyk K, Rojewska E, Przewlocka B. Importance of glial activation in neuropathic pain. Eur J Pharmacol 2013; 716:106-119. 
44. Ledeboer A, Sloane EM, Milligan ED, Frank MG, Mahony JH, Maier SF, Watkins LR. Minocycline attenuates mechanical allodynia and proinflammatory cytokine expression in rat models of pain facilitation. Pain 2005; 115:71-83.

45. Chye CL, Liang CL, Lu K, Chen YW, Liliang PC. Pulsed radiofrequency treatment of articular branches of femoral and obturator nerves for chronic hip pain. Clin Interv Aging 2015; 10:569-574.

46. Wan CF, Liu Y, Dong DS, Zhao L, Xi Q, Yu X, Cui WY, Wang QS, Song T. Bipolar High-Voltage, Long-Duration Pulsed radiofrequency improves pain relief in postherpetic neuralgia. Pain Physician 2016; 19:E721-728.

47. Luo F, Lu J, Shen Y, Meng L, Wang T, Ji N. Effectiveness and safety of pulsed ra- diofrequency treatment guided by computed tomography for refractory neuralgia of Infraorbital nerve: A pilot study. Pain Physician 2015; 18 E795-804.

48. Choi S, Choi HJ, Cheong Y, Lim YJ, Park HK. Internal-specific morphological analysis of sciatic nerve fibers in a radiofrequency-induced animal neuropathic pain model. PLoS One 2013; 8:e73913. 